\title{
Meaning of reaction orders in polymer:fullerene solar cells
}

\author{
Thomas Kirchartz* and Jenny Nelson \\ Department of Physics and Centre for Plastic Electronics, Imperial College London, South Kensington Campus, \\ London SW7 2AZ, United Kingdom
}

(Received 10 August 2012; revised manuscript received 12 September 2012; published 5 October 2012)

\begin{abstract}
Nongeminate recombination in polymer:fullerene solar cells is frequently characterized using transient optoelectronic measurements that allow the determination of recombination rates, charge carrier lifetimes, and average charge carrier concentrations as a function of voltage. These data are often interpreted in terms of an empirical reaction order defining how recombination depends on measured charge density. In polymer:fullerene solar cells, the empirical reaction orders are often considerably larger than 2, which had previously been explained in terms of the nonlinear relationship between mobile and trapped charge carriers in the presence of an exponential tail of localized states. Here, we show that experimentally determined reaction orders depend not only on the shape of the density of states but also on the spatial distribution of carriers. In particular, in solar cells with small depletion regions due to small active layer thicknesses or due to large unintentional background doping of the polymers, the reaction order can assume values that are much larger than the value expected from the shape of the density of states alone.
\end{abstract}

DOI: 10.1103/PhysRevB.86.165201

\section{INTRODUCTION}

The main electronic loss mechanism in inorganic thin-film solar cells is nongeminate recombination of two initially separated charge carriers. Due to the low dielectric constants, and in consequence due to the high exciton binding energies in molecular semiconductors, the situation is less clear in organic solar cells where geminate recombination of excitons or bound charge carriers can be a substantial loss mechanism. ${ }^{1,2}$ However, recent studies ${ }^{3-13}$ suggest that nongeminate recombination is limiting the efficiency of most well-performing bulk heterojunction solar cells. Experimentally, nongeminate recombination is often studied at the open circuit where, due to the lack of an external current flow, effects of series resistances and charge carrier transport are minimized. Methods such as the transient photovoltage measurement allow the determination of charge carrier lifetimes or recombination rates as a function of open-circuit voltage. Combined with measurements of the charge carrier density derived from the integration of current transients, the obtained lifetimes and recombination rates are often presented as functions of the charge density at the open circuit and a reaction order is defined. ${ }^{14-16}$ The empirical reaction order $\delta$ describes how the recombination rate $R$ scales with the charge carrier concentration $n_{\mathrm{CE}}$ that is obtained by integrating the transient current resulting from switching off the light bias on a solar cell and short circuiting the device at the same time. The relationship between recombination rate and charge concentration at the open circuit follows a power law according to

$$
R\left(n, p, V_{\mathrm{oc}}\right) \propto n_{\mathrm{CE}}^{\delta}\left(V_{\mathrm{oc}}\right) .
$$

In addition to this empirical definition, we could use a conceptual definition of the reaction order. If we define the reaction order $\Delta$ in terms of free carriers as $\Delta=$ $d \log (R) / d \log \left(n_{\mathrm{CE}}\right)$, we obtain a quantity that has a more straightforward relationship to the recombination mechanism than does $\delta$. If a free electron is recombining with a deeply trapped hole, the reaction order $\Delta$ would be 1 , because the concentration of deeply trapped holes doesn't change with
PACS number(s): 73.61.Ph, 84.60.Jt, 72.20.Jv, 72.80.Ng

voltage and illumination. If two free carriers are recombining, $\Delta=2$. Thus, $\Delta$ should vary between 1 and 2 in the absence of Auger-like processes, and the closer $\Delta$ is to 1 , the deeper and more dominant are the traps involved in recombination. However, in practice, the empirical reaction orders $\delta$ are often substantially higher than $2^{17,18}$ and sometimes have values above $5 .{ }^{19}$ These experimental observations make the reaction order rather difficult to understand and require a closer understanding of the differences between $\delta$ and $\Delta$.

A widely used explanation for empirical reaction orders $\delta$ higher than 2 is the presence of energetic disorder leading to a broad distribution of electronic states that are active in recombination to different degrees. ${ }^{7,18,20,21}$ This distribution of electronic states leads to a discrepancy between the reaction order $\Delta$ in terms of free carriers and the reaction order $\delta$ in terms of all measured carriers. In the classical multiple trapping theory used in modeling of both organic ${ }^{7,22}$ and inorganic ${ }^{23,24}$ solar cells based on disordered absorber materials, any recombination event involves at least one free charge carrier, while the second charge carrier might be sitting in a shallow trap in a band tail state. Assuming that the carriers in the band tails will still be extracted during the measurement, only part of the extracted electrons recombines with (all) holes, and vice versa. Because the ratio of free to trapped carriers increases with overall charge density, the reaction order is larger than 2 , with the exact value depending on the shape of the density of tail states. ${ }^{7,20}$ However, the quantitative interpretation of average, experimentally determined charge concentrations in terms of a density of localized states relies on the assumption of a spatially homogeneous excess carrier density.

In this article, we review how to understand reaction orders in cases in which the energy-dependent density of states and the recombination mechanism define the reaction orders, as discussed earlier. Subsequently, we discuss how the spatial distribution of charge carriers affects the reaction order, which is the more relevant the thinner the active layer is. Therefore, most polymer:fullerene solar cells with active layer thicknesses below $100 \mathrm{~nm}$ are strongly affected by the 
spatial distribution of electrons and holes, and an interpretation in terms of their energetic distribution, i.e., a density of trap states, is challenging or impossible.

We discuss why a solid understanding of the reaction order requires us to first study the dependence of recombination current on voltage, defined by the so-called ideality factor, as well as the relationships between charge density and voltage and charge lifetime and voltage. We explain the relationships among recombination rate, carrier density, and voltage in a consistent way. We also discuss the influence of different types of localized states, different thicknesses, different doping concentrations, and different types of recombination on the experimentally accessible quantities.

\section{DESCRIPTION OF THE EXPERIMENTS TO BE SIMULATED}

To understand experimentally determined reaction orders and their relationship to the internal properties of the device, like the density of localized states, we use steady-state driftdiffusion simulations (as described, e.g., in Refs. 7, 9, and 23) to simulate three experiments that are routinely performed on polymer:fullerene solar cells by different groups. $5,16,17,25-30$ The simulations are based on solving the Poisson equation connecting the electrostatic potential with the space charge and the continuity equations for electrons and holes on a one-dimensional grid. The conduction band of the simulation represents the lowest unoccupied molecular orbital of the fullerene, and the valence band of the simulation represents the highest occupied molecular orbital (HOMO) of the polymer. To take the disordered nature of the device into account we use a multiple trapping model that incorporates trapping and detrapping of charges in tail states that are exponentially distributed in energy (Sec. III) and Shockley-Read-Hall ${ }^{31,32}$ recombination between free charges and charges trapped in these tail states. In later sections of this paper, we add recombination via deep states $^{12,13}$ (Sec. IV) and surface recombination $^{33}$ (Sec. V) of charge carriers at cathode or anode to the model. In addition, we add charge due to doping ${ }^{34}$ (Sec. VII) to the space charge and discuss the influence of this additional charge on the spatial distribution of carriers and the interpretation of experimental data. The model is identical ${ }^{35,36}$ or similar ${ }^{37,38}$ to models used in the past for amorphous $\mathrm{Si}$ solar cells.

The measurements we simulate with this model are the light intensity-dependent open-circuit voltage measurement, the charge extraction measurement, and the transient photovoltage measurement. In the following, we introduce the empirical relationships that are derived from the experiments.

\section{A. Ideality factor}

The open-circuit voltage $V_{\text {oc }}$ depends in theory-and nearly always in practice - on the logarithm of the light intensity. It can be described by

$$
V_{\mathrm{oc}}=n_{\mathrm{id}} \frac{k T}{q} \ln (\phi)+\text { const. }
$$

where $k T$ is the thermal energy, $q$ is the elementary charge, $\phi$ is the incident photon flux, and $n_{\text {id }}$ is the ideality factor. The ideality factor is indicative of the recombination mechanism and is usually in the range between 1 and 2, with values toward 2 indicating recombination via deeper states. ${ }^{39}$ Because at the open circuit the average generation and average recombination rates have to cancel out to guarantee zero net current flow, and because the generation rate is proportional to the incident light intensity, Eq. (2) implies that the average recombination rate $R$ at the open circuit scales as $R=R_{0} \exp \left(q V_{\mathrm{oc}} / n_{\mathrm{id}} k T\right)$, with $R_{0}$ as a proportionality factor. In only a few cases ${ }^{40}$ does the opencircuit voltage $V_{\mathrm{oc}}$ saturate at higher light intensities (i.e., $n_{\mathrm{id}}<$ 1 and strongly voltage dependent), which is likely an indication of increased surface recombination due to $V_{\text {oc }}$ approaching the built-in voltage. ${ }^{20} \mathrm{We}$ use Eq. (2) as a definition for $n_{\mathrm{id}}$ rather than the more common definition of $n_{\text {id }}$ via the slope of the dark current-voltage curve, because in experiments the dark current is often strongly affected by shunt and series resistances. Because the series resistance is irrelevant at the open circuit (zero current flow, i.e., zero voltage drop over a series resistance), using the light intensity dependence of $V_{\mathrm{oc}}$ is more favorable.

To determine $n_{\text {id }}$ from experimentation, a straight line is usually fitted to the $V_{\text {oc }}$ plotted vs the logarithm of the light intensity. Alternatively, the derivative $n_{\mathrm{id}}=$ $(q / k T)\left(d V_{\mathrm{oc}} / d \ln \phi\right)$ is calculated as a function of $V_{\mathrm{oc}}$ or light intensity and is then averaged. In the simulation, we first calculate $V_{\text {oc }}$ for different light intensities and then calculate the derivative of the slope and average to obtain the ideality factor $n_{\text {id }}$.

\section{B. Charge extraction}

During a charge extraction experiment, ${ }^{25}$ a solar cell is held at a certain light and voltage bias before the light bias is switched off at the same time as the voltage is switched to short circuit. The charge carriers that were electrically and optically injected into the solar cell are now extracted, and the extracted charge density (in As $/ \mathrm{cm}^{2}$ ) $Q_{\mathrm{CE}}=q n_{\mathrm{CE}} d$, derived from the integral over the current transient, is interpreted in terms of an average carrier concentration $n_{\mathrm{CE}}$ if the active layer thickness $d$ is known. Thus, in the ideal case of efficient extraction, the charge extraction method measures the difference in charge carrier concentration at a given light and voltage bias relative to short circuit in the dark. Thus, the measured charge density $n_{\mathrm{CE}}$ is

$$
n_{\mathrm{CE}}=\frac{1}{d} \int_{0}^{d} n(V, \phi, x)-n\left(0, \phi_{\mathrm{bb}}, x\right) d x+\frac{\Delta \sigma_{\mathrm{cath}}(V, \phi)}{q d} .
$$

Here, $x$ is the spatial coordinate normal to the device surface, $d$ is the active layer thickness, and $\phi_{\mathrm{bb}}$ is the black body photon flux of the environment, i.e., the thermal radiation that is present in the dark at room temperature. In general, the charge density $\sigma$ on the electrodes changes as a function of light and voltage bias, and because of the mobile charges in the device, it does so in a nonlinear way. Thus, the measured charge density $n_{\mathrm{CE}}$ can always be affected by the charge on the electrodes rather than in the active layer. Thus, the active layer charge has to be corrected by the change $\Delta \sigma_{\text {cath }}$ in the charge density on the cathode (if the first term on the right-hand side of Eq. (3) is written in terms of electron density). This is 
particularly important in case of doping of the active layer, as discussed in more detail in Sec. VII.

We use the definition in Eq. (3) for our simulations, but in practice the charge density $n_{\mathrm{CE}}$ might be reduced due to recombination at short circuit relative to the ideal case for devices with low mobilities and lifetimes or large active layer thicknesses. The typical dependence of the charge density $n_{\mathrm{CE}}$ on internal voltage $V$ is

$$
n_{\mathrm{CE}}=n_{0, \mathrm{CE}} \exp \left(\frac{q V}{m k T}\right),
$$

where the constant $m$ specifies how the slope deviates from the thermal voltage $k T / q$.

\section{Transient photovoltage}

The last measurement is the transient photovoltage experiment, where the device is kept at the open circuit at a certain bias light intensity. A small additional light pulse creates an increase of $V_{\mathrm{oc}}$ that decays exponentially back to the background value after the end of the light pulse. A transient photovoltage measurement is usually performed at a series of different light intensities so that the lifetime can be plotted vs the open-circuit voltage. The decay of the excess open-circuit voltage is proportional to the decay of excess charges if done in the small perturbation regime. ${ }^{25}$ Subsequently, the small signal lifetime from transient photovoltage measurements can be used to compute a large signal lifetime $\tau_{\mathrm{n}}{ }^{41}$ that is the ratio of excess carrier concentration and recombination rate $R\left(\tau=n_{\mathrm{CE}} / R\right)$. Because we know how charge density and recombination rate depend on the open-circuit voltage, it follows that

$$
\begin{aligned}
\tau_{\mathrm{n}} & =\frac{n_{\mathrm{CE}}}{R}=\frac{n_{0, \mathrm{CE}}}{R_{0}} \exp \left(\frac{q V_{\mathrm{oc}}}{k T}\left[\frac{1}{m}-\frac{1}{n_{\mathrm{id}}}\right]\right) \\
& \equiv \tau_{0} \exp \left(-\frac{q V_{\mathrm{oc}}}{\vartheta k T}\right),
\end{aligned}
$$

where we introduced the abbreviations $\tau_{0}$ for the extrapolated lifetime at $V_{\mathrm{oc}}=0$ and $\vartheta=\left(n_{\mathrm{id}}^{-1}-m^{-1}\right)^{-1}$ for the slope. Because $m>n_{\mathrm{id}}, \vartheta>0$ in all practical cases, implying that in an organic bulk heterojunction solar cell, the lifetime decays exponentially with increasing open-circuit voltage. This is equivalent to saying that the measured reaction order is always larger than 1, i.e., that recombination increases faster with voltage than the carrier concentration does.

\section{Empirical reaction order}

There are two ways to determine the reaction order $\delta$ experimentally from the measurements discussed earlier. One option is to plot the carrier concentration determined by charge extraction vs the recombination current. The recombination current can be derived from the dark current density; alternatively, the light intensity corresponding to a certain open-circuit voltage can be use, because it must be proportional to the recombination rate by the requirement of zero net current flow at the open circuit. Another alternative is to calculate the recombination rate using the electron density $n_{\mathrm{CE}}$ determined by charge extraction, the transient photovoltage lifetime $\tau$, and $R=n_{\mathrm{CE}} / \tau$ [Eq. (5)], which usually leads to nearly the same result. ${ }^{16,17}$ In our simulations, the lifetime is calculated from Eq. (5); thus, the two ways to determine the reaction order are mathematically identical, and the empirical reaction order can be written as

$$
\delta=\frac{d \ln (R)}{d \ln \left(n_{\mathrm{CE}}\right)}=\frac{d V}{d \ln \left(n_{\mathrm{CE}}\right)} \frac{d \ln (R)}{d V}=\frac{m}{n_{\mathrm{id}}},
$$

i.e., as a function of the slopes $m$ and $n_{\text {id }}$ only. Equation (6) is central to the paper, because it implies that all information contained in the reaction order can be explained by studying the behavior of the ideality factor $n_{\text {id }}$ and the voltage dependence of the charge density (determined by $m$ ).

To understand how to interpret the slope $\vartheta$ and the reaction order $\delta$, we have to study how the spatial and energetic distributions of carriers affect the way the extracted charge scales with internal voltage affecting $m$ and how different recombination mechanisms affect $n_{\text {id }}$. In the following, we therefore discuss different recombination mechanisms and their influence on $m$ and $n_{\text {id }}$ before we discuss how changes in the spatial carrier distributions, as induced by changes in thickness or doping concentration, affect $m$. To facilitate the understanding of the recombination mechanisms on the experimental results, we attempt to derive analytical approximations for symmetric situations first and use them to discuss the general trends. In cases in which simple analytical approximations do not exist, we rely on numerical simulations.

\section{RECOMBINATION VIA BAND TAILS}

In an intrinsic, field-free region of a perfectly crystalline semiconductor, electron and hole concentrations should each scale with $n=p \propto \exp (q V / 2 k T)$. This assumes that carriers are delocalized in a conduction or valence band and the Fermi level is sufficiently far $(>3 k T)$ from the edge of the band to allow the Boltzmann approximation to be used. Organic solar cells are made from disordered materials that contain a large amount of localized states below the actual transport energy, which is typically called mobility edge ${ }^{42}$ in disordered semiconductors. In this case, the Boltzmann approximation no longer properly describes the voltage dependence of the concentration of electrons and holes. ${ }^{43}$ Instead, the density of localized states as a function of energy determines the slope $m$. We assume here for simplicity exponential band tails as shown in Fig. 1(a), which seem to be a decent approximation to experimental results..$^{8,11,22,44}$ We define the density of states as

$$
N_{\mathrm{CBT}}=N_{0 \mathrm{CBT}} \exp \left(\frac{E-E_{\mathrm{C}}}{E_{\mathrm{chC}}}\right)
$$

for the conduction band tail and

$$
N_{\mathrm{VBT}}=N_{0 \mathrm{VBT}} \exp \left(-\frac{E-E_{\mathrm{V}}}{E_{\mathrm{chV}}}\right)
$$

for the valence band tail. Here, $E_{\mathrm{C}}$ and $E_{\mathrm{V}}$ are the conduction band edge and the valence band edge [cf. Fig. 1(a)], respectively, that separate the mobile states from the immobile, trapped states. The tail slopes are $E_{\mathrm{chC}}$ and $E_{\mathrm{chV}}$, and the absolute concentration of tail states is controlled by the prefactors $N_{0 \mathrm{CBT}}$ and $N_{0 \mathrm{VBT}}$, which define the concentration of states per volume and energy interval at $E_{\mathrm{C}}$ and $E_{\mathrm{V}}$. 


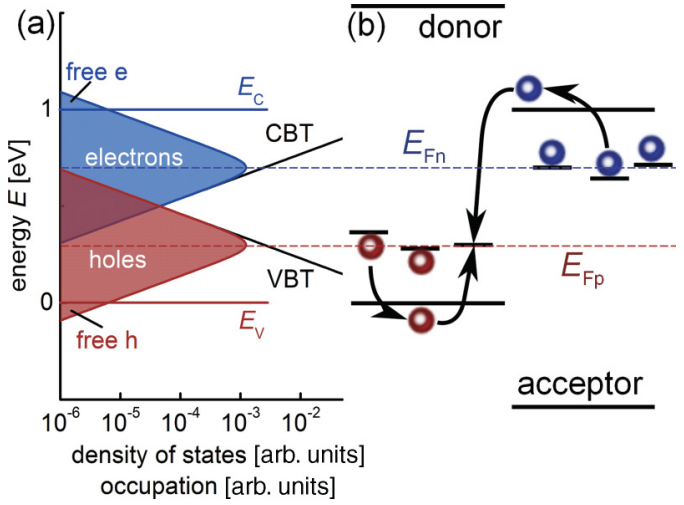

FIG. 1. (Color online) Schematic depiction of (a) the density of states and occupation in a system with exponential tails and (b) the recombination via tail states. The occupation of a tail with a slope $E_{\text {ch }}>k T$ is always such that most carriers are trapped close to the quasi-Fermi levels (dashed lines). Thus, a recombination event requires one of the trapped carriers to become mobile and recombine with the other carrier trapped close to the interface at the respective quasi-Fermi level. CBT, conduction band tail; VBT, valence band tail.

If the tail slope of a band is larger than $k T$, the electrons or holes mostly occupy states close to the quasi-Fermi level, as shown in Figs. 1(a) and 1(b), ${ }^{43}$ but their mobility may not be sufficiently high to allow transport to happen via these states. Whether recombination happens via states below the mobility edge is debated. A considerable number of publications have suggested that these tail states could affect the recombination. ${ }^{6,7,44-48}$ While we think that tail-to-tail recombination is probably not favorable due the lack of hopping sites at the same energy, we think it is likely that recombination happens between a free carrier and one carrier trapped in a band tail [as shown in Fig. 1(a)], which would be consistent with ideality factors higher than 1 seen in materials like P3HT:PCBM. 6,7,20,49

The concentration of trapped charge carriers in a system with two identical tails $\left(E_{\mathrm{chC}}=E_{\mathrm{chV}}=E_{\mathrm{ch}}\right)$ scales approximately as ${ }^{7,23,24,31,32,35,51}$ (see also the Supplemental Material ${ }^{50}$ )

$$
n_{\mathrm{t}}=p_{\mathrm{t}} \propto \exp \left(\frac{\Delta E_{\mathrm{f}}}{2 E_{\mathrm{ch}}}\right),
$$

with the quasi-Fermi level splitting $\Delta E_{\mathrm{f}}=E_{\mathrm{fn}}-E_{\mathrm{fp}}$. The general idea to derive Eq. (8) is to assume that the band tails defined in Eq. (7) are filled up to the energies $E_{\mathrm{fn}}$ or $E_{\mathrm{fp}}$ and that the occupation probability can be approximated by a step function that is 1 below $E_{\mathrm{fn}}$ and 0 above $E_{\mathrm{fn}}$ (or 1 above $E_{\mathrm{fp}}$ and 0 below $E_{\mathrm{fp}}$ ).

If we are able to probe the internal voltage, e.g., by measuring at the open circuit, the charge extraction scales according to Eq. (4) with the factor $m$ given by

$$
m=\frac{2 E_{\mathrm{ch}}}{k T} .
$$

In the Shockley-Read-Hall model, recombination takes place between free and trapped carriers. That means the recombina- tion rate scales with

$$
\begin{aligned}
R & \propto n p_{\mathrm{t}} \propto \exp \left(\frac{q V}{2 k T}\right) \exp \left(\frac{q V}{2 E_{\mathrm{ch}}}\right) \\
& =\exp \left(\frac{q V}{k T}\left[\frac{1}{2}+\frac{k T}{2 E_{\mathrm{ch}}}\right]\right),
\end{aligned}
$$

leading to an ideality factor as derived by van Berkel et $a l .{ }^{39}$ :

$$
n_{\mathrm{id}}=\left(\frac{1}{2}+\frac{k T}{2 E_{\mathrm{ch}}}\right)^{-1} \text {. }
$$

Now, we can directly use Eqs. (5) and (6) to derive the reaction order

$$
\delta=\frac{m}{n_{\mathrm{id}}}=\frac{E_{\mathrm{ch}}}{k T}+1
$$

and the slope

$$
\vartheta=\left(n_{\mathrm{id}}^{-1}-m^{-1}\right)^{-1}=2
$$

of lifetime vs open-circuit voltage.

To test our zero-dimensional analytical approximations, we compare Eqs. (9) and (11)-(13) with drift-diffusion simulations using a multiple trapping model as described in Refs. 7 and 23 and the parameters detailed in Table I. Figure 2(a) shows the results for the ideality factor as a function of tail slope $E_{\mathrm{ch}}$. In this simulation, all parameters have been chosen to be equal for electrons and holes, including the tail slope. The analytical approximation [Eq. (11)] nicely follows the numerical simulation for tail slopes $E_{\text {ch }}>k T$, while the ideality factor approaches 1 for shallower tail slopes $E_{\mathrm{ch}}<k T$. Figure 2(b) compares the analytical approximations for the factors $m$ (for $\log (n)$ vs $V_{\mathrm{oc}}$ ), $\delta$ (for the reaction order), and $\vartheta$ (for the slope of $\log (\tau)$ vs $V_{\mathrm{oc}}$ ) with numerical simulations. Again, agreement is good for $E_{\mathrm{ch}}>k T$, although $m$ and $\delta$ are slightly overestimated by the analytical approximations for higher tail slopes. This is because it starts to matter that a charge extraction experiment measures the carrier concentration at a given $V_{\mathrm{oc}}$ with respect to the carrier concentration at $0 \mathrm{~V}$ in the dark, which is not necessarily negligible for broad tails.

It is reasonable to assume, however, that the tail slopes are not necessarily identical. To study that scenario, we repeated the simulations but kept one tail slope constant and varied the other while keeping the total concentration of trap states the same. Now, the splitting of the quasi-Fermi levels is no longer symmetric around midgap because the total charge has to stay 0 . So to keep $n=p$ at any given voltage,

$$
\begin{aligned}
n & =p \propto \exp \left(\frac{q V}{m k T}\right)=\exp \left(\frac{E_{\mathrm{fn}}-E_{\mathrm{i}}}{E_{\mathrm{chC}}}\right) \\
& =\exp \left(\frac{E_{\mathrm{i}}-E_{\mathrm{fp}}}{E_{\mathrm{chV}}}\right)
\end{aligned}
$$

would have to be fulfilled in a zero-dimensional approximation, i.e., if we assume that all charge carrier concentrations are constant as a function of position. Here, $E_{\mathrm{i}}$ is the intrinsic Fermi level, i.e., midgap. In Eq. (14), we assumed again that both tail slopes are broader than $k T$, as for all our analytical approximations before. After a short calculation that uses $q V \approx E_{\mathrm{fn}}-E_{\mathrm{fp}}$, we arrive at

$$
m=\frac{E_{\mathrm{chC}}+E_{\mathrm{chV}}}{k T}
$$


TABLE I. Parameters used for the simulations, presented in Figs. 2-5, 7, and 8. The mobilities given are band mobilities and apply only to the untrapped fraction of the charge carrier population. For the parameters (capture coefficient $\beta_{\mathrm{ds}}$, total density $N_{\mathrm{ds}}$, Gaussian width $\sigma_{\mathrm{ds}}$ of the distribution, and correlation energy $U$ ) used for the recombination via deep defects, see the Supplemental Material for exact definitions (Refs. 50 and 55).

\begin{tabular}{|c|c|c|c|c|}
\hline & Tail recombination & Tail + deep & Surface recombination & Doping and thickness series \\
\hline Fig. no. & 2,3 & 4 & 5 & 8,10 \\
\hline$\mu_{\mathrm{n}}\left(\mathrm{cm}^{2} / \mathrm{Vs}\right)$ & $10^{-3}$ & $10^{-3}$ & Variable & $10^{-3}$ \\
\hline$\mu_{\mathrm{p}}\left(\mathrm{cm}^{2} / \mathrm{Vs}\right)$ & $10^{-3}$ & $10^{-3}$ & Variable & $10^{-3}$ \\
\hline$N_{\mathrm{C}}\left(\mathrm{cm}^{-3}\right)$ & $10^{19}$ & $10^{19}$ & $10^{19}$ & $10^{19}$ \\
\hline$N_{\mathrm{V}}\left(\mathrm{cm}^{-3}\right)$ & $10^{19}$ & $10^{19}$ & $10^{19}$ & $10^{19}$ \\
\hline$N_{\text {Ctail }}\left(\mathrm{cm}^{-3}\right)$ & $2.5 \times 10^{19}$ & $2.5 \times 10^{18}$ & $2.5 \times 10^{18}$ & $2.5 \times 10^{18}$ \\
\hline$N_{\text {Vtail }}\left(\mathrm{cm}^{-3}\right)$ & $2.5 \times 10^{19}$ & $2.5 \times 10^{18}$ & $2.5 \times 10^{18}$ & $2.5 \times 10^{18}$ \\
\hline$E_{\mathrm{chC}}(\mathrm{meV})$ & Variable & 50 & 50 & 50 \\
\hline$E_{\mathrm{chV}}(\mathrm{meV})$ & Variable & 50 & 50 & 50 \\
\hline$\beta_{\mathrm{n}}+\left(\mathrm{cm}^{3} \mathrm{~s}^{-1}\right)$ & $10^{-12}$ & $10^{-12}$ & $10^{-12}$ & $10^{-12}$ \\
\hline$\beta_{\mathrm{p}}{ }^{0}\left(\mathrm{~cm}^{3} \mathrm{~s}^{-1}\right)$ & $10^{-10}$ & $10^{-10}$ & $10^{-10}$ & $10^{-10}$ \\
\hline$\beta_{\mathrm{p}}-\left(\mathrm{cm}^{3} \mathrm{~s}^{-1}\right)$ & $10^{-12}$ & $10^{-12}$ & $10^{-12}$ & $10^{-12}$ \\
\hline$\beta_{\mathrm{n}}^{0}\left(\mathrm{~cm}^{3} \mathrm{~s}^{-1}\right)$ & $10^{-10}$ & $10^{-10}$ & $10^{-10}$ & $10^{-10}$ \\
\hline$E_{\mathrm{g}}(\mathrm{eV})$ & 1.1 & 1.1 & 1.1 & 1.1 \\
\hline$d(\mathrm{~nm})$ & 150 & 150 & 150 & 150 and variable \\
\hline$S\left(\mathrm{~cm} \mathrm{~s}^{-1}\right)$ & 0 & 0 & $10^{5}$ & 0 \\
\hline$\varphi_{\mathrm{b}}(\mathrm{meV})$ & 0.1 & 0.1 & 0.1 & 0.1 \\
\hline$G\left(\mathrm{~cm}^{-3} \mathrm{~s}^{-1}\right)$ & $4 \times 10^{21}$ & $4 \times 10^{21}$ & $4 \times 10^{21}$ & $4 \times 10^{21}$ \\
\hline$\beta_{\mathrm{ds}}\left(\mathrm{cm}^{3} \mathrm{~s}^{-1}\right)$ & 0 & $10^{-10}$ & 0 & $10^{-10}$ \\
\hline$N_{\mathrm{ds}}\left(\mathrm{cm}^{-3}\right)$ & 0 & Variable & 0 & $10^{16}$ \\
\hline$\sigma_{\mathrm{ds}}(\mathrm{eV})$ & 0 & 0.15 & 0 & 0.15 \\
\hline$U(\mathrm{eV})$ & 0 & 0 & 0 & 0 \\
\hline
\end{tabular}

i.e., the average tail slope determines the increase of carrier concentration with quasi-Fermi level splitting in the case of asymmetric tail slopes. Figure 3(b) shows that Eq. (15) is a

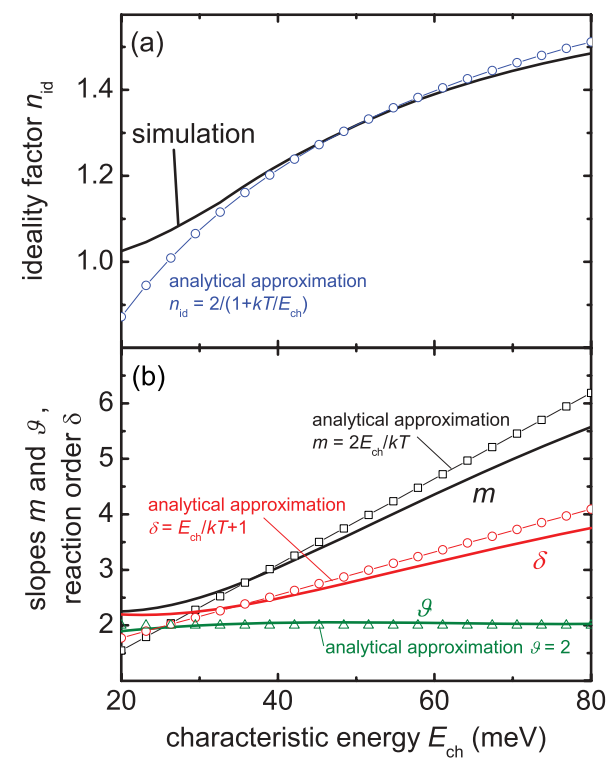

FIG. 2. (Color online) Simulated dependence of (a) ideality factor $n_{\text {id }}$ and (b) slope $m$ (defined by $\left.n_{\mathrm{CE}} \sim \exp \left(q V_{\mathrm{oc}} / m k T\right)\right)$, reaction order $\delta$, and slope $\vartheta$ (defined by $\tau=\tau_{0} \exp \left(-q V_{\text {oc }} / \vartheta k T\right)$ ) on tail slope $E_{\mathrm{ch}}$, assuming that recombination happens via conduction and valence band tail states (solid lines). The symbols represent the analytical approximations that hold for $E_{\mathrm{ch}}>k T$ and are defined in Eqs. (9) and (11)-(13). reasonable approximation for the lower characteristic energies and overestimates the slope $m$ at higher tail slopes, indicating that in this case, a zero-dimensional model cannot capture all the physics in a one-dimensional numerical model.

To derive a similar analytical approximation for the ideality factor is rather difficult, because we would need to know not only how the total concentration of electrons and holes scales with voltage but also how the individual concentrations of free and trapped electrons behave. From Fig. 3(a), we see that the numerical simulation empirically follows a small modification of the original van Berkel equation, which replaces the tail slope with the maximum value of the two tail slopes, i.e.,

$$
n_{\mathrm{id}}=\left(\frac{1}{2}+\frac{k T}{2 \max \left(E_{\mathrm{chC}}, E_{\mathrm{chV}}\right)}\right)^{-1}
$$

Consequently, the reaction order is deduced from the intuitive Eq. (15) and from Eq. (16), which is based on the observation of the numerical trends, as

$$
\delta=\frac{m}{n_{\mathrm{id}}}=\frac{\min \left(E_{\mathrm{chC}}, E_{\mathrm{chV}}\right)}{k T}+\frac{\max \left(E_{\mathrm{chC}}, E_{\mathrm{chV}}\right)}{2 k T} .
$$

This means that the reaction order is dominated by the smaller of the two tail slopes while the ideality factor is dominated by the larger of the two tail slopes. Comparing the numerical simulations with Eq. (17) in Fig. 3(b) shows that the second term on the right-hand side in Eq. (17) is mostly suppressed in the numerical simulation. 


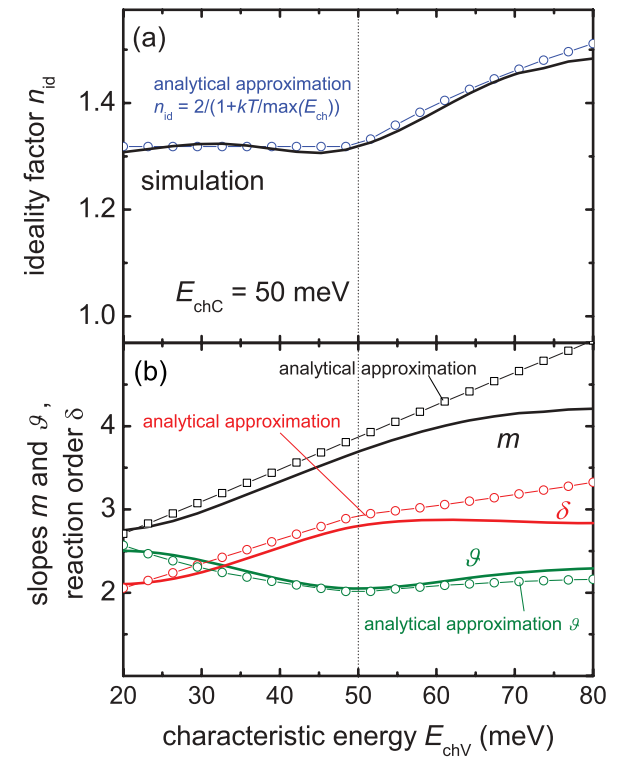

FIG. 3. (Color online) Simulated dependence of (a) $n_{\text {id }}$ and (b) $m$, $\delta$, and $\vartheta$ on the valence band tail slope, assuming that the conduction band tail slope is fixed at $E_{\mathrm{chC}}=50 \mathrm{meV}$ (solid lines). Again, analytical approximations are shown that work well only in a limited range of characteristic energies. From the simulations, we conclude that the ideality factor $n_{\text {id }}$ is mostly determined by the larger tail slope, while the reaction order $\delta$ is dominated by the smaller tail slope.

\section{RECOMBINATION VIA DEEP STATES}

In the case of recombination via tail states, we assume that recombination takes place via the same population of trapped electrons and holes in the band tail that we detect in charge extraction measurements. However, it is possible that recombination involves traps that are energetically too deep to be visible in charge extraction measurements. In this case, the information on the density of states we obtain from charge extraction would be decoupled from the information on the density of states we get from the ideality factor. Charge extraction would be sensitive to the shallow traps (e.g., tail states) that can still be extracted, while recombination would be sensitive to the deep states, which we define as those states at which charge cannot be collected in a charge extraction experiment. Shockley-Read-Hall recombination via deep states at the donor-acceptor interface was recently suggested as a possible limitation for the open-circuit voltage and as a reason for the high ideality factor in different polymer:fullerene solar cells. ${ }^{4,8,11,52,53}$ Street et al. recently presented experimental evidence for the formation of deep states after irradiation of polymer:fullerene devices with $\mathrm{x}$ rays ${ }^{13}$ and white light, as well as by annealing of devices. ${ }^{12}$

Again, we perform simulations with the parameters given in column 2 of Table I and using the Sah-Shockley statistics ${ }^{54}$ for the occupation statistics of the deep states (see the Supplemental Material ${ }^{55}$ ). In contrast to the earlier simulations, we include a recombination via a Gaussian distribution of states centered at midgap; we also reduce the concentration of tails to keep the effect of the traps on a similar level as before and to better study the effects of the additional deep traps. In addition, the charge density that we now use to calculate $m$ and $\delta$ is only

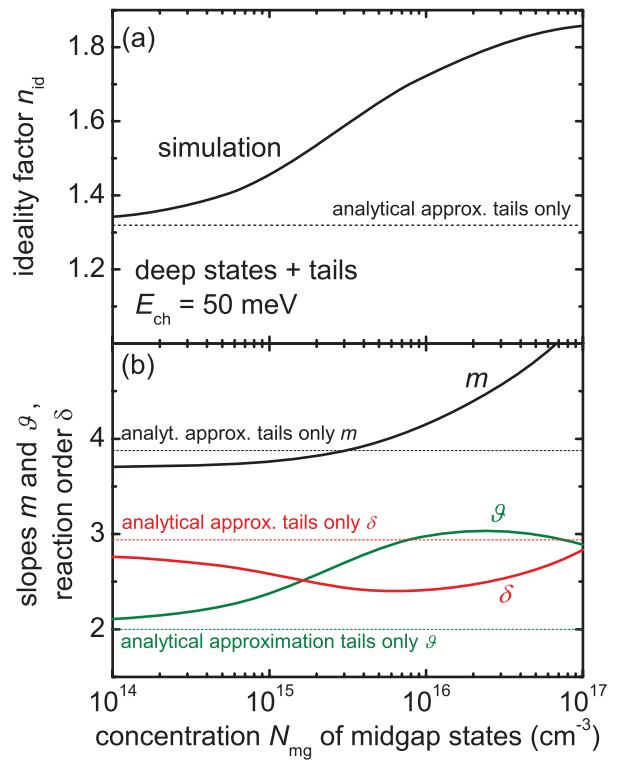

FIG. 4. (Color online) Simulated dependence of (a) $n_{\mathrm{id}}$ and (b) $m, \delta$, and $\vartheta$ on the concentration of midgap states, assuming that recombination happens both via tails and via deep states (solid lines). The simulation shows a transition from the situation with tail recombination dominating to a situation that is dominated by recombination via deep states, mainly resulting in a change of $n_{\text {id }}$ rather than $m$. High concentrations of deep states therefore lead to smaller reaction orders $\delta=m / n_{\text {id }}$ and a high value of $\vartheta$. The thin dashed lines represent the analytical approximations assuming just symmetric tail states with slope $E_{\mathrm{chC}}=50 \mathrm{meV}$.

the charge density in the tail states, while we assume that the charge in the deep states is invisible during a charge extraction experiment.

Figure 4 shows the effect of increasing the concentration of deep traps. For low concentrations of deep states, we obtain the result for tail recombination only with $E_{\mathrm{chC}}=E_{\mathrm{chV}}=E_{\mathrm{ch}}=$ $50 \mathrm{meV}$. For higher concentrations of traps, the ideality factor increases drastically, reflecting that most recombination is now via the deep traps. A midgap trap should lead to an ideality factor of 2 when $n=p$, but due to the combination of midgap and tail recombination, as well as due to the inhomogeneity of the carrier concentrations, we instead obtain a continuous transition from a value of $\sim 1.3$ (for tail recombination only) to nearly 2 for a large concentration of deep traps. In contrast, the voltage dependence of the carrier concentration $m$ is only weakly affected by the change in deep state concentration. The reaction order $\delta=m / n_{\text {id }}$ varies only marginally, while $\vartheta=$ $\left(n_{\mathrm{id}}^{-1}-m^{-1}\right)^{-1}$ increases slightly for larger concentrations of deep states.

\section{RECOMBINATION AT THE CONTACTS}

In addition to recombination at the internal donor-acceptor interface, recombination might happen at the interfaces to the contact layers, typically PEDOT:PSS at the anode and a metal layer at the cathode. This type of recombination-usually called surface recombination - is one of the main loss mechanisms in crystalline Si solar cells; therefore, the passivation of the surfaces of the silicon wafer is a major concern in this 
field. ${ }^{56-58}$ In the context of organic solar cells, surface recombination is rarely discussed in much detail, and there are only few theoretical studies on its effects. ${ }^{9,33,59-61}$ However, there are extensive studies on cathode interlayers, ${ }^{62-64}$ electrode work functions, ${ }^{65-68}$ and hole transporting layers ${ }^{69-71}$ and their effect on the device performance, including the open-circuit voltage. All of these studies implicitly support the theory that, at least in some organic solar cells, recombination at the contact affects the open-circuit voltage.

Surface recombination is controlled by the concentration of excess minority carriers $\Delta n_{\min }$ at the cathode or anode and the surface recombination velocity $S_{\min }$ via

$$
J_{\mathrm{S}}=q S_{\min } \Delta n_{\min } .
$$

The surface recombination velocity is proportional to the concentration of surface states that are active in recombination and their capture cross section, and it is in principle possible that $S_{\text {min }}$ depends on $\Delta n_{\text {min }}$, making surface recombination nonlinear. However, at present, we assume $S_{\min }$ to be constant. The excess electron (hole) concentration at the anode (cathode) depends primarily on how easy it is for the electron (hole) to diffuse against the built-in electric field to the anode (cathode). Thus, device thickness, mobility, and built-in voltage are the parameters that affect surface recombination, in addition to the value of the surface recombination velocity. We assume that the capture coefficients for any recombination events at the internal donor-acceptor interface are independent of mobility. Thus, at the open circuit, the only process limited by mobility is the diffusion of electrons to the anode and holes to the cathode, which means that the fraction of surface recombination increases with mobility.

Figure 5 shows a simulation using a variation in charge carrier mobility to study the effect of surface recombination on the voltage dependence of carrier concentration and recombination. With increasing mobility, increases occur in the concentration of the minorities at the contacts and therefore the relative influence of surface recombination. At the cathode $n \gg p$ holds, while at the anode $p \gg n$; i.e., at the contacts the minority carrier type is well defined. This is a good approximation as long as the open-circuit voltage $V_{\mathrm{oc}}$ is much smaller $\left(V_{\mathrm{bi}}-V_{\mathrm{oc}} \gg k T\right)$ than the built-in voltage $V_{\mathrm{bi}}$, which is the case for the simulations presented here and is likely the case for optimized practical organic solar cells. In the case discussed here, where $n \gg p$ or $p \gg n$ holds at the contacts, any increase in quasi-Fermi level splitting $\Delta E_{f}$ (by applying a voltage or increasing the light intensity) would lead to an increase in the minority carrier concentration and keep the majority carrier concentration constant. Thus, surface recombination usually has an ideality factor of 1 , because it is directly proportional to the excess minority carrier concentration at the contact that scales with $\Delta n_{\min } \propto \exp \left(\Delta E_{\mathrm{f}} / k T\right)$. Because no current is flowing at $V_{\mathrm{oc}}$, there is no or little variation in the quasi-Fermi levels over the device thickness, and $q V_{\mathrm{oc}} \approx \Delta E_{f}$. This explains why the ideality factor in Fig. 5 decreases with increasing mobility and approaches 1 at high mobilities where surface recombination is the dominant recombination mechanism. Again, the voltage dependence $m$ of carrier concentration is only weakly affected by the change in mobility, while $\delta$ and $\vartheta$ now show the opposite trend, as

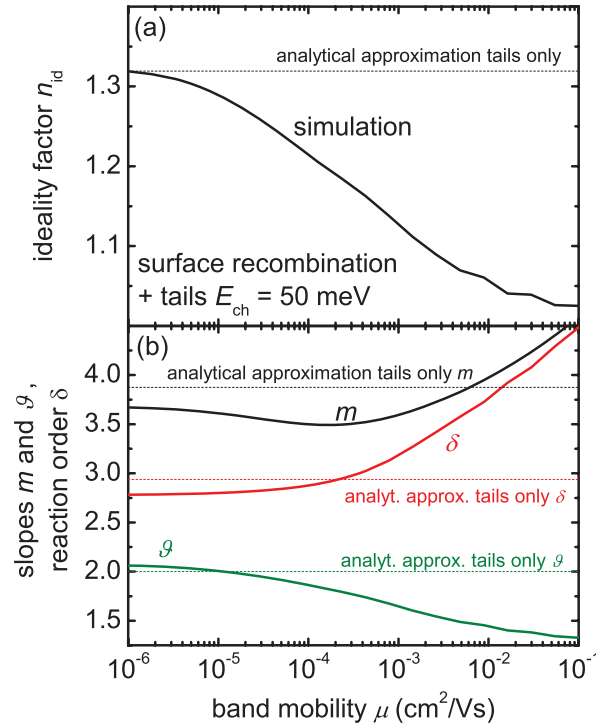

FIG. 5. (Color online) Simulated dependence of (a) $n_{\text {id }}$ and (b) $m, \delta$, and $\vartheta$ on the mobility of both carriers, assuming that electrons can recombine at the anode and holes can recombine at the cathode (surface recombination). For higher mobilities, the relative importance of surface recombination increases, leading essentially to a smaller ideality factor $n_{\mathrm{id}}$. The thin dashed lines represent the analytical approximations assuming just symmetric tail states with slope $E_{\mathrm{chC}}=50 \mathrm{meV}$.

in the case of deep states. Because the ideality decreases with increasing mobility and $m$ even slightly increases, the reaction order increases drastically when recombination is limited by surface recombination. In contrast, the slope $\vartheta$ decreases below the values calculated earlier for recombination via traps at the internal donor-acceptor heterointerface. The result for surface recombination applies to other recombination mechanisms as well, because recombination between free electrons and holes gives low ideality factors in devices where traps still exist that are visible in charge extraction and that raise $m$ above 2 .

\section{THE INFLUENCE OF THE SPATIAL CARRIER DISTRIBUTION}

So far, we have dealt with the influence of the concentration of electrons and holes as a function of energy on the charge extraction result and the influence of different recombination mechanisms on the voltage dependence of recombination rate and carrier lifetime. However, there are cases in which the spatial dependence of the carrier concentration ${ }^{72}$ dominates the voltage dependence of the carrier concentration. The most obvious case is when the device thickness is reduced below $100 \mathrm{~nm}$. For typical polymer:fullerene solar cells, the first absorption maximum is at a thickness of $\sim 80 \mathrm{~nm},{ }^{73,74}$ and due to low mobilities, the optimum thickness is at the first absorption maximum rather than at the second. Therefore, the investigation of the effect of low thicknesses is important in particular for materials other than the few polymers like P3HT, which are typically fabricated with active layer thicknesses $d \gg 100 \mathrm{~nm}^{75,76}$ 


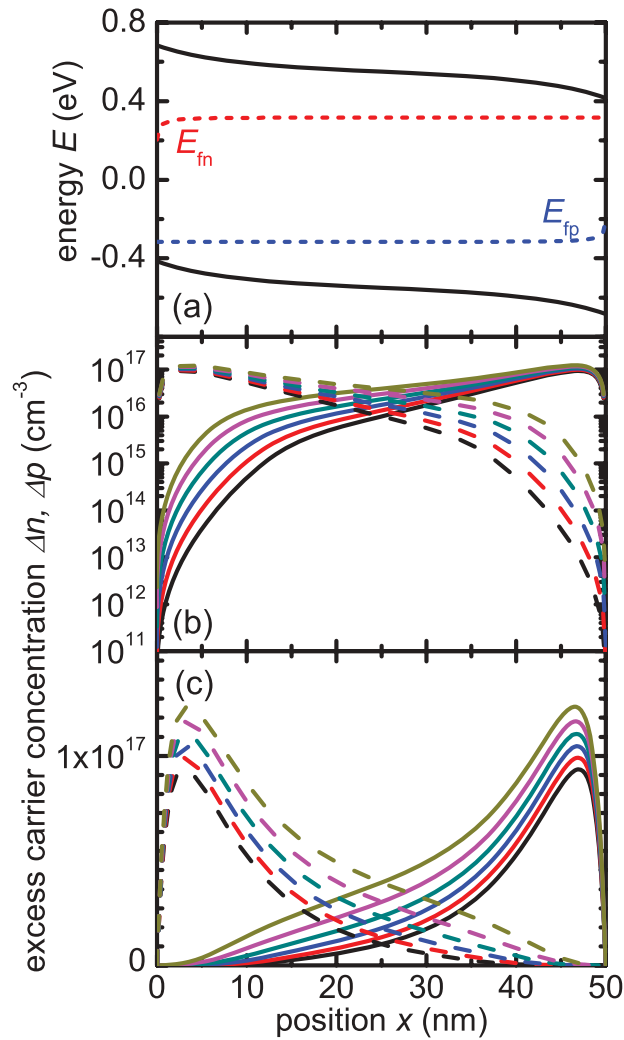

FIG. 6. (Color online) (a) Band diagram, as well as (b) and (c) electron and hole concentration as a function of position for a 50-nm-thick active layer. The excess electron and hole concentrations are depicted for different light intensities, always at the open circuit, and the band diagram corresponds to the highest light intensity. The excess electron and hole concentrations are spatially inhomogeneous, which leads to a slow increase of the average carrier concentration with voltage because most change happens where the carrier concentrations are low, which contributes only slightly to the average carrier concentration.

Figure 6 shows the simulated (a) band diagram and (b) and (c) excess carrier concentration of a 50-nm thin solar cell at the open circuit for different photogeneration rates. In the band diagram, the conduction band edge $E_{\mathrm{C}}$ represents the mobility edge of the fullerene and the valence band edge $E_{\mathrm{V}}$ represents the mobility edge in the polymer. The quasi-Fermi levels for electrons and holes are mostly flat because at the open circuit, the current flow is 0 ; therefore, the gradient of the quasi-Fermi levels of the majority carriers must be 0 as well at the contacts that extract the majority carriers. At the opposite contact, surface recombination leads to a gradient of the quasi-Fermi level of the minority carrier. However, the bands are not flat, because the built-in voltage is assumed to be larger than the open-circuit voltage to reduce surface recombination. Thus, the carrier concentrations are also not flat but rather follow $n(x) \sim \exp \left[\left(E_{C}-E_{\mathrm{fn}}\right) /(m(x) k T)\right]$ and $p(x) \sim \exp \left[\left(E_{\mathrm{fp}}-E_{\mathrm{V}}\right) /(m(x) k T)\right]$, where $m(x)$ is a locally defined quantity as opposed to the global $m$ discussed in the rest of the article. Therefore, the inhomogeneous electron concentration, e.g., increases with voltage rapidly close to the anode (where $n \ll p$ ) and slowly close to the cathode. However, the integrated carrier concentration is now dominated by the

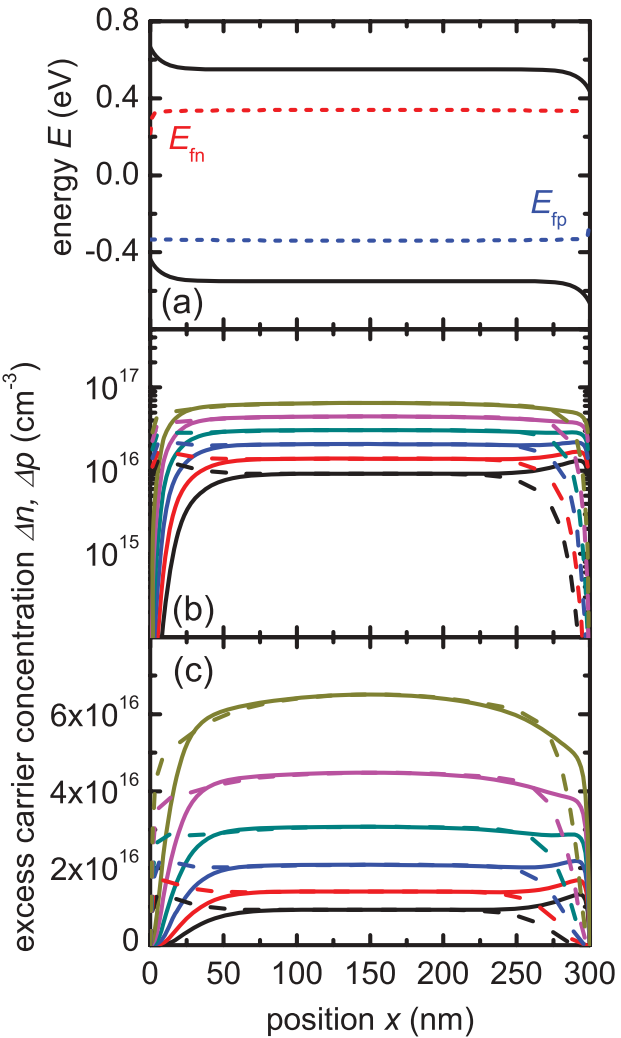

FIG. 7. (Color online) (a) Band diagram, as well as (b) and (c) electron and hole concentration as a function of position for a 300-nm-thick active layer. The excess electron and hole concentrations are depicted for different light intensities, always at the open circuit, and the band diagram corresponds to the highest light intensity. In contrast to the case of a thin solar cell (Fig. 6), the carrier concentration is spatially homogeneous and is well described by analytical models based on one-dimensional approximations.

concentration close to the cathode, as can be seen in Fig. 6(c). Thus, in inhomogeneous carrier concentrations, the integrated carrier concentration increases more slowly with voltage than in homogeneous carrier concentrations. Figure 7 shows the band diagram and the carrier concentrations for the case of a thick cell in which the space charge regions at the contacts are small compared to the cell thickness; therefore, a large part of the device is field free, and the excess carrier concentration is nearly homogeneous.

Figure 8 summarizes the effect of thickness on the voltage dependence of average carrier concentration and carrier lifetime. In terms of the recombination mechanism, a thin cell is more likely to be affected by surface recombination than a thick cell, because the diffusion toward the wrong electrode is easier. We chose the parameters for the recombination mechanism such that Fig. 8(a) shows this transition from low $n_{\text {id }}$ at low thickness (surface recombination dominates) to larger $n_{\mathrm{id}}$ at high thickness (recombination via deep states dominates). Figure 8(b) shows that the average carrier concentration increases very slowly with voltage at low thicknesses $d$, as explained earlier ( $m$ becomes large at low $d$ ). Thus, we can now obtain massively high reaction orders above 6 in thin devices with $d<70 \mathrm{~nm}$. Therefore, the empirical reaction order, as defined by Eq. (1), is difficult to interpret, being a complicated 


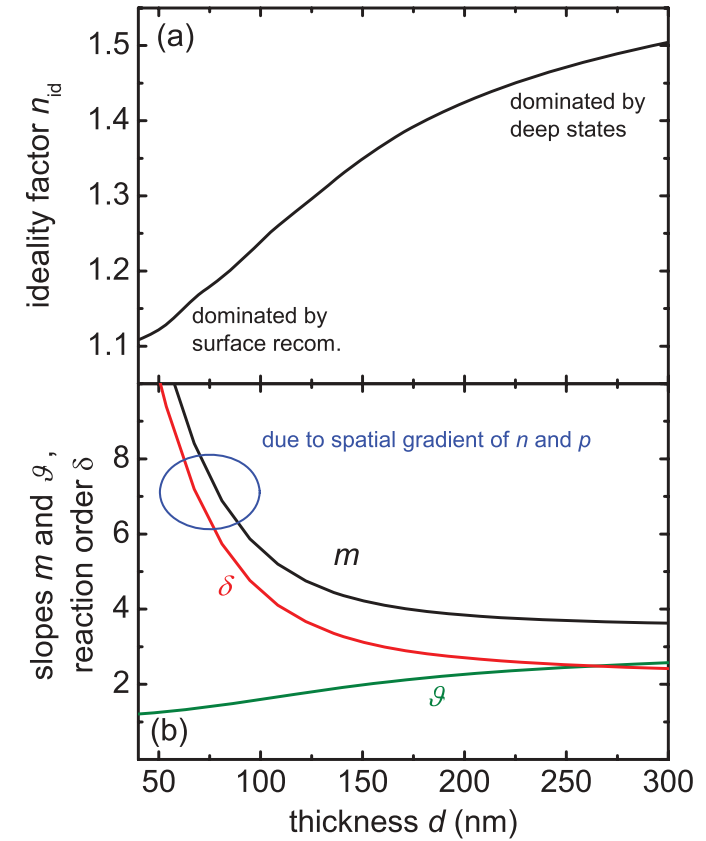

FIG. 8. (Color online) Simulated dependence of (a) $n_{\text {id }}$ and (b) $m, \delta$, and $\vartheta$ as a function of active layer thickness. The device is assumed to be dominated by recombination via deep states if thick. When the thickness is reduced, it becomes easier for the charge carriers to reach the opposite electrode and surface recombination becomes dominant, reducing the ideality factor. The slope $m$ and the reaction order increase strongly at low thicknesses because of the spatial inhomogeneity of the carrier concentration, as shown in Fig. 6.

function of recombination mechanism and carrier distribution. A normal comparison between, e.g., a 200-nm-thick solar cell and a 100-nm-thick solar cell from another polymer:fullerene blend would inevitably lead to drastically different reaction orders ${ }^{18}$ that could be misinterpreted in terms of fundamentally different physics.

\section{INFLUENCE OF DOPING}

Studies of compensation experiments ${ }^{34}$ or capacitance voltage measurements of polythiophene:fullerene solar cells suggest that the blend layer is not necessarily perfectly intrinsic, as is often assumed in models of device functionality, but is instead $p$-type. ${ }^{77-81}$ Although the potential effect of doping is limited for thin devices, where the space charge region created by the doping is larger than the cell thickness, it may be of high relevance to devices with higher thicknesses and higher doping concentrations. ${ }^{81}$ In doped devices, we observe two important differences to the intrinsic situation discussed earlier. First, depending on the doping concentration, the active layer is not charge neutral if the charge on the contact layers is not taken into account. It is therefore not possible to distinguish the active layer charge from the charge on the electrodes. Thus, $\int \Delta n d x \neq \int \Delta p d x$ and the final term in Eq. (3) become highly relevant.

The second main issue with doped devices is that the majority carrier concentration is relatively homogenous over the thickness of the device, because it is given by the doping concentration in the bulk and space charge regions form

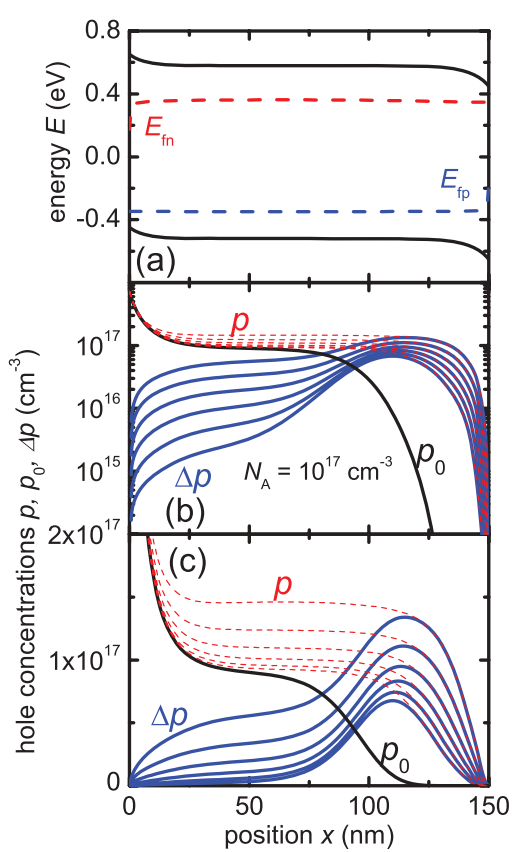

FIG. 9. (Color online) (a) Band diagram, as well as (b) and (c) hole concentration as a function of position for a 150-nm-thick active layer with $p$-type doping. Panels (b) and (c) depicts the hole concentrations $p$, the concentration at $0 \mathrm{~V}$ in the dark $p_{0}$, and the excess concentration $\Delta p=p-p_{0}$. As for the case of a thin solar cell, the excess carrier concentration is spatially inhomogeneous, although the total concentration $p$ is rather homogeneous.

only near the contacts. However, charge extraction detects excess carrier concentration, which is highly inhomogeneous. This may be counterintuitive, but it becomes obvious when considering that in the bulk, the photogeneration does not greatly change the majority carrier concentration. Instead, the majority carrier concentration stays roughly constant at the value given by the doping concentration until, at higher light intensities, the excess photogenerated carriers exceed the doping concentration. The main change in carrier concentration takes place where the equilibrium carrier concentration is not that high, i.e., at the edge of the space charge region to the metal back contact, and is therefore highly inhomogeneous. A graphical representation of that effect is shown in Fig. 9. In essence, the effect of doping is similar to the effect of thin cells essentially because the space charge region is thin in both cases. In terms of recombination, doping creates an asymmetry between electrons and holes. In the case of $p$-type doping, e.g., there are far fewer electrons than holes throughout most of the thickness of the device. In this case, recombination scales with the minority carrier concentration and the ideality factor is close to 1 , independent of the energetic distribution of defect states [Fig. 10(a)].

At low light intensities and voltages, the change in excess carrier concentration is small, and it gets larger only at higher light intensities. Thus, the slope $m$ increases drastically with higher doping concentrations [Fig. 10(b)]. Both the increase of $m$ and the decrease of $n_{\text {id }}$ push the empirical reaction order $\delta=m / n_{\text {id }}$ up with increasing doping concentration. For the parameters chosen here, the slope $\vartheta$ is slightly below 3 at low doping densities and then slowly decreases to values 


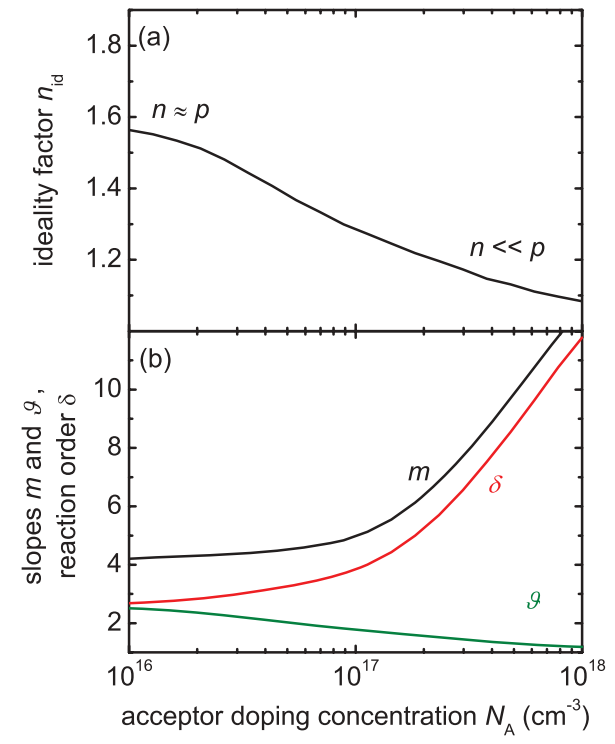

FIG. 10. (Color online) Simulated dependence of (a) $n_{\text {id }}$ and (b) $m, \delta$, and $\vartheta$ as a function of the concentration of effective acceptor doping. For a higher doping concentration, recombination depends only on the concentration of free minority carriers (here, electrons). The ideality factor therefore drops to 1 , independent of the density of localized states. Because doping leads to a spatially inhomogeneous distribution of excess carriers, at high doping concentrations, the slope $m$ and therefore the reaction order $\delta$ increase drastically.

close to 2 for higher doping concentrations. Essentially, the behavior of all four slopes is analogous to the reduction in device thickness. The more spatially inhomogeneous the excess carrier concentration (the lower the thickness, the higher the doping concentration), the higher the empirical reaction order and the higher the parameter $m$ that controls how the carrier concentration scales with voltage.

\section{CONCLUSIONS}

We discussed the interdependence of the different slopes that are obtained from plotting recombination current, carrier concentration, voltage, and charge carrier lifetime against one another, showing that all information is contained in just two numbers, the diode ideality factor $n_{\text {id }}$ and the slope $m$ characterizing the dependence of excess carrier concentration on voltage. Both quantities mainly depend on (1) the density of states and (2) the excess carrier distribution in space. We first discussed idealized situations in which the excess carrier concentration is homogeneous enough to allow the density of states to dominate the behavior of the device. Using numerical simulations and analytical approximations, we showed how the shape of the density of shallow or deep localized states affects $n_{\text {id }}$ and $m$. In this situation, the empirical reaction order is a measure of the density of states only. However, if we proceed to situations in which the excess carrier concentrations are spatially inhomogeneous, the ideality factor continues to remain a useful indication of the recombination mechanism, while all other slopes start to reflect the distribution of the carrier concentration in space rather than in energy. For active layer thicknesses below $100 \mathrm{~nm}$, the empirical reaction order starts to increase strongly due to the large gradient in carrier concentration. Thus, an interpretation of changing reaction order in terms of a change in recombination mechanism is not possible in these cases. For thicker solar cells, typical doping concentrations observed in capacitance voltage experiments lead to a similar inhomogeneity in excess carrier concentration and to high empirical reaction orders.

\section{ACKNOWLEDGMENTS}

T.K. acknowledges support by an Imperial College Junior Research Fellowship, and J.N. acknowledges support from a Royal Society Industrial Fellowship. The authors thank the engineering and physical sciences research council for support, especially via the Supergen Excitonic Solar Cell Consortium (EP/G031088/1).

\section{APPENDIX: THE REACTION ORDER IN TERMS OF FREE CARRIERS}

The reaction order as determined from charge extraction experiments is often not an intuitive quantity for people who are used to defining reaction orders in terms of the free carrier population that follows a Boltzmann distribution, because it exists in states that are more than $3 k T$ away from the quasi-Fermi level. Let us therefore discuss how reaction orders $\Delta$ in terms of free carriers compare with reaction orders $\delta$ in terms of free carriers and charges trapped in tail states. Let us consider a case with symmetric tail slopes in a zero-dimensional approximation so that $n_{\mathrm{f}}=p_{\mathrm{f}}$ and $n_{t}=p_{t}$. We allow recombination between free electrons and trapped holes, as well as between free holes and trapped electrons, and neglect recombination of free electrons with free holes. Then, we can write the recombination rate as

$$
R \propto n_{\mathrm{f}} n_{\mathrm{t}}=\frac{n_{\mathrm{f}}}{n_{\mathrm{t}}} n_{\mathrm{t}}^{2}=\frac{n_{\mathrm{t}}}{n_{\mathrm{f}}} n_{\mathrm{f}}^{2} .
$$

Using $n_{\mathrm{f}}=n_{\mathrm{t}}^{\left(\frac{E_{\mathrm{ch}}}{k T}\right)}$, we can express the recombination rate either as a function of free electron concentration $n_{\mathrm{f}}$ or as a function of the electron concentration $n_{\mathrm{t}}$ in tail states, i.e.,

$$
R \propto n_{\mathrm{f}} n_{\mathrm{t}}=n_{\mathrm{t}}^{\left(\frac{E_{\mathrm{ch}}}{\mathrm{kT}}+1\right)}=n_{\mathrm{f}}^{\left(\frac{\mathrm{kT}}{E_{\mathrm{ch}}}+1\right)} .
$$

From Eq. (A2), we see that the deeper the traps (the higher $\left.E_{\mathrm{ch}}\right)$, the higher the reaction order in terms of trapped carriers ( $\left.\delta=E_{\mathrm{ch}} / k T+1\right)$ but the lower the reaction order in terms of free carriers $\left(\Delta=k T / E_{\mathrm{ch}}+1\right)$. For large tail slopes, the reaction order as measured with charge extraction would be large and the reaction order in terms of free carriers would be 1 . Thus, the term monomolecular, which has been used for recombination via traps, can make sense for large $E_{\mathrm{ch}}$ when the reaction order is discussed in terms of free carriers. However, because this reaction order is difficult or impossible to measure, recombination via traps has been often labeled as bimolecular in the literature, ${ }^{16}$ which has led to confusion about the terminology. ${ }^{82,83}$ 
*t.kirchartz@imperial.ac.uk

${ }^{1}$ J. A. Barker, C. M. Ramsdale, and N. C. Greenham, Phys. Rev. B 67, 075205 (2003).

${ }^{2}$ L. J. A. Koster, E. C. P. Smits, V. D. Mihailetchi, and P. W. M. Blom, Phys. Rev. B 72, 085205 (2005).

${ }^{3}$ R. A. Street, S. Cowan, and A. J. Heeger, Phys. Rev. B 82, 121301 (2010).

${ }^{4}$ R. A. Street, M. Schoendorf, A. Roy, and J. H. Lee, Phys. Rev. B 81, 205307 (2010).

${ }^{5}$ C. G. Shuttle, R. Hamilton, B. C. O'Regan, J. Nelson, and J. R. Durrant, Proc. Natl. Acad. Sci. USA 107, 16448 (2010).

${ }^{6}$ R. C. I. MacKenzie, T. Kirchartz, G. F. A. Dibb, and J. Nelson, J. Phys. Chem. C 115, 9806 (2011).

${ }^{7}$ T. Kirchartz, B. E. Pieters, J. Kirkpatrick, U. Rau, and J. Nelson, Phys. Rev. B 83, 115209 (2011).

${ }^{8}$ R. A. Street, K. W. Song, J. E. Northrup, and S. Cowan, Phys. Rev. B 83, 165207 (2011).

${ }^{9}$ S. Schafer, A. Petersen, T. A. Wagner, R. Kniprath, D. Lingenfelser, A. Zen, T. Kirchartz, B. Zimmermann, U. Wurfel, X. J. Feng, and T. Mayer, Phys. Rev. B 83, 165311 (2011).

${ }^{10}$ G. F. A. Dibb, T. Kirchartz, D. Credgington, J. R. Durrant, and J. Nelson, J. Phys. Chem. Lett. 2, 2407 (2011).

${ }^{11}$ R. A. Street, Phys. Rev. B 84, 075208 (2011).

${ }^{12}$ R. A. Street, A. Krakaris, and S. R. Cowan, Adv. Funct. Mater., doi: 10.1002/adfm.201200031 (2012).

${ }^{13}$ R. A. Street, J. E. Northrup, and B. S. Krusor, Phys. Rev. B 85, 205211 (2012).

${ }^{14}$ G. Juska, K. Genevicius, N. Nekrasas, G. Sliauzys, and G. Dennler, Appl. Phys. Lett. 93, 143303 (2008).

${ }^{15}$ C. Deibel, A. Baumann, and V. Dyakonov, Appl. Phys. Lett. 93, 163303 (2008).

${ }^{16}$ C. G. Shuttle, B. O’Regan, A. M. Ballantyne, J. Nelson, D. D. C. Bradley, and J. R. Durrant, Phys. Rev. B 78, 113201 (2008).

${ }^{17}$ A. Maurano, R. Hamilton, C. G. Shuttle, A. M. Ballantyne, J. Nelson, B. O'Regan, W. M. Zhang, I. McCulloch, H. Azimi, M. Morana, C. J. Brabec, and J. R. Durrant, Adv. Mater. 22, 4987 (2010).

${ }^{18}$ D. Rauh, C. Deibel, and V. Dyakonov, Adv. Funct. Mater. 22, 3371 (2012).

${ }^{19}$ D. Credgington and J. R. Durrant, J. Phys. Chem. Lett. 3, 1465 (2012).

${ }^{20}$ J. C. Blakesley and D. Neher, Phys. Rev. B 84, 075210 (2011).

${ }^{21}$ A. Foertig, J. Rauh, V. Dyakonov, and C. Deibel, Phys. Rev. B 86, 115302 (2012).

${ }^{22}$ R. C. I. MacKenzie, C. G. Shuttle, M. L. Chabinyc, and J. Nelson, Adv. Energ. Mater. 2, 662 (2012).

${ }^{23}$ B. E. Pieters, K. Decock, M. Burgelman, R. Stangl, and T. Kirchartz, in Advanced Characterization Techniques for Thin Film Solar Cells (Wiley-VCH Verlag GmbH \& Co. KGaA, Weinheim, Germany, 2011), pp. 501.

${ }^{24}$ B. E. Pieters, T. Kirchartz, T. Merdzhanova, and R. Carius, Sol. Energ. Mater. Sol. Cell. 94, 1851 (2010).

${ }^{25}$ C. G. Shuttle, B. O'Regan, A. M. Ballantyne, J. Nelson, D. D. C. Bradley, J. de Mello, and J. R. Durrant, Appl. Phys. Lett. 92, 093311 (2008).

${ }^{26}$ C. G. Shuttle, A. Maurano, R. Hamilton, B. O’Regan, J. C. de Mello, and J. R. Durrant, Appl. Phys. Lett. 93, 183501 (2008).
${ }^{27}$ A. Maurano, C. C. Shuttle, R. Hamilton, A. M. Ballantyne, J. Nelson, W. M. Zhang, M. Heeney, and J. R. Durrant, J. Phys. Chem. C 115, 5947 (2011).

${ }^{28}$ A. Sanchez-Diaz, M. Izquierdo, S. Filippone, N. Martin, and E. Palomares, Adv. Funct. Mater. 20, 2695 (2010).

${ }^{29}$ D. Rauh, A. Wagenpfahl, C. Deibel, and V. Dyakonov, Appl. Phys. Lett. 98, 133301 (2011).

${ }^{30}$ D. Credgington, R. Hamilton, P. Atienzar, J. Nelson, and J. R. Durrant, Adv. Funct. Mater. 21, 2744 (2011).

${ }^{31}$ W. Shockley and W. T. Read, Phys. Rev. 87, 835 (1952).

${ }^{32}$ R. N. Hall, Phys. Rev. 87, 387 (1952).

${ }^{33}$ T. Kirchartz, B. E. Pieters, K. Taretto, and U. Rau, Phys. Rev. B 80, 035334 (2009).

${ }^{34}$ Z. Q. Liang and B. A. Gregg, Adv. Mater. 24, 3258 (2012).

${ }^{35}$ B. E. Pieters, Characterization of Thin-Film Silicon Materials and Solar Cells through Numerical Modeling (Ph.D. Thesis, Delft University of Technology, Delft, the Netherlands, 2008), pp. 32, http://repository.tudelft.nl/view/ir/uuid:83bc5ff4-8e33-4962-901468cfbad8226f/.

${ }^{36}$ M. Zeman and J. Krc, J. Mater. Res. 23, 889 (2008).

${ }^{37}$ M. Hack and M. Shur, J. Appl. Phys. 58, 997 (1985).

${ }^{38}$ P. J. Mcelheny, P. Chatterjie, and S. J. Fonash, J. Appl. Phys. 69, 7674 (1991).

${ }^{39}$ C. Vanberkel, M. J. Powell, A. R. Franklin, and I. D. French, J. Appl. Phys. 73, 5264 (1993).

${ }^{40}$ Y. Zhang, X. D. Dang, C. Kim, and T. Q. Nguyen, Adv. Energ. Mater. 1, 610 (2011).

${ }^{41}$ B. C. O'Regan, J. R. Durrant, P. M. Sommeling, and N. J. Bakker, J. Phys. Chem. C 111, 14001 (2007).

${ }^{42}$ B. E. Pieters, H. Stiebig, M. Zeman, and R. A. C. M. van Swaaij, J. Appl. Phys. 105, 044502 (2009).

${ }^{43}$ W. Gong, M. A. Faist, N. J. Ekins-Daukes, Z. Xu, D. D. C. Bradley, J. Nelson, and T. Kirchartz, Phys. Rev. B 86, 024201 (2012).

${ }^{44}$ J. Nelson, Phys. Rev. B 67, 155209 (2003).

${ }^{45}$ M. Tachiya and K. Seki, Phys. Rev. B 82, 085201 (2010).

${ }^{46}$ M. P. Eng, P. R. F. Barnes, and J. R. Durrant, J. Phys. Chem. Lett. 1, 3096 (2010).

${ }^{47}$ G. Garcia-Belmonte and J. Bisquert, Appl. Phys. Lett. 96, 113301 (2010).

${ }^{48}$ N. C. Giebink, G. P. Wiederrecht, M. R. Wasielewski, and S. R. Forrest, Phys. Rev. B 82, 155305 (2010).

${ }^{49}$ G. A. H. Wetzelaer, M. Kuik, M. Lenes, and P. W. M. Blom, Appl. Phys. Lett. 99, 153506 (2011).

${ }^{50}$ See Supplemental Material at http://link.aps.org/supplemental/ 10.1103/PhysRevB.86.165201 for a derivation of Eq. (8) (Sec. II).

${ }^{51}$ J. G. Simmons and G. W. Taylor, Phys. Rev. B 4, 502 (1971).

${ }^{52}$ S. R. Cowan, W. L. Leong, N. Banerji, G. Dennler, and A. J. Heeger, Adv. Funct. Mater. 21, 3083 (2011).

${ }^{53}$ W. L. Leong, S. R. Cowan, and A. J. Heeger, Adv. Energ. Mater. 1, 517 (2011).

${ }^{54}$ C.-T. Sah and W. Shockley, Phys. Rev. 109, 1103 (1958).

${ }^{55}$ See Supplemental Material at http://link.aps.org/supplemental/ 10.1103/PhysRevB.86.165201 for more details on the model used for recombination via deep states (Sec. I).

${ }^{56}$ B. Hoex, J. Schmidt, P. Pohl, M. C. M. van de Sanden, and W. M. M. Kessels, J. Appl. Phys. 104, 044903 (2008).

${ }^{57}$ B. Hoex, J. Schmidt, R. Bock, P. P. Altermatt, M. C. M. van de Sanden, and W. M. M. Kessels, Appl. Phys. Lett. 91, 112107 (2007). 
${ }^{58}$ J. Schmidt, A. Merkle, R. Brendel, B. Hoex, M. C. M. van de Sanden, and W. M. M. Kessels, Prog. Photovoltaics 16, 461 (2008).

${ }^{59}$ J. C. Scott and G. G. Malliaras, Chem. Phys. Lett. 299, 115 (1999).

${ }^{60}$ T. Kirchartz, B. E. Pieters, K. Taretto, and U. Rau, J. Appl. Phys. 104, 094513 (2008).

${ }^{61}$ A. Wagenpfahl, C. Deibel, and V. Dyakonov, IEEE J. Sel. Top. Quant. Electron. 16, 1759 (2010).

${ }^{62}$ J. H. Seo, A. Gutacker, Y. M. Sun, H. B. Wu, F. Huang, Y. Cao, U. Scherf, A. J. Heeger, and G. C. Bazan, J. Am. Chem. Soc. 133, 8416 (2011).

${ }^{63}$ Z. C. He, C. M. Zhong, X. Huang, W. Y. Wong, H. B. Wu, L. W. Chen, S. J. Su, and Y. Cao, Adv. Mater. 23, 4636 (2011).

${ }^{64}$ K. Asadi, P. de Bruyn, P. W. M. Blom, and D. M. de Leeuw, Appl. Phys. Lett. 98, 183301 (2011).

${ }^{65}$ C. J. Brabec, A. Cravino, D. Meissner, N. S. Sariciftci, T. Fromherz, M. T. Rispens, L. Sanchez, and J. C. Hummelen, Adv. Funct. Mater. 11, 374 (2001).

${ }^{66}$ V. D. Mihailetchi, L. J. A. Koster, and P. W. M. Blom, Appl. Phys. Lett. 85, 970 (2004).

${ }^{67}$ V. D. Mihailetchi, P. W. M. Blom, J. C. Hummelen, and M. T. Rispens, J. Appl. Phys. 94, 6849 (2003).

${ }^{68}$ A. Guerrero, L. F. Marchesi, P. P. Boix, S. Ruiz-Raga, T. RipollesSanchis, G. Garcia-Belmonte, and J. Bisquert, ACS Nano 6, 3453 (2012).

${ }^{69}$ K. X. Steirer, P. F. Ndione, N. E. Widjonarko, M. T. Lloyd, J. Meyer, E. L. Ratcliff, A. Kahn, N. R. Armstrong, C. J. Curtis, D. S. Ginley, J. J. Berry, and D. C. Olson, Adv. Energ. Mater. 1, 813 (2011).
${ }^{70}$ M. D. Irwin, B. Buchholz, A. W. Hains, R. P. H. Chang, and T. J. Marks, Proc. Natl. Acad. Sci. USA 105, 2783 (2008).

${ }^{71}$ E. L. Ratcliff, B. Zacher, and N. R. Armstrong, J. Phys. Chem. Lett. 2, 1337 (2011).

${ }^{72}$ C. Deibel, A. Wagenpfahl, and V. Dyakonov, Phys. Rev. B 80, 075203 (2009).

${ }^{73}$ H. Hoppe, N. Arnold, N. S. Sariciftci, and D. Meissner, Sol. Energ. Mater. Sol. Cell. 80, 105 (2003).

${ }^{74}$ F. Monestier, J. J. Simon, P. Torchio, L. Escoubas, F. Florya, S. Bailly, R. de Bettignies, S. Guillerez, and C. Defranoux, Sol. Energ. Mater. Sol. Cell. 91, 405 (2007).

${ }^{75}$ J. Peet, L. Wen, P. Byrne, S. Rodman, K. Forberich, Y. Shao, N. Drolet, R. Gaudiana, G. Dennler, and D. Waller, Appl. Phys. Lett. 98, 043301 (2011)

${ }^{76}$ S. C. Price, A. C. Stuart, L. Q. Yang, H. X. Zhou, and W. You, J. Am. Chem. Soc. 133, 4625 (2011).

${ }^{77}$ J. Bisquert and G. Garcia-Belmonte, J. Phys. Chem. Lett. 2, 1950 (2011).

${ }^{78}$ F. Fabregat-Santiago, G. Garcia-Belmonte, I. Mora-Sero, and J. Bisquert, Phys. Chem. Chem. Phys. 13, 9083 (2011).

${ }^{79}$ J. Bisquert, G. Garcia-Belmonte, A. Munar, M. Sessolo, A. Soriano, and H. J. Bolink, Chem. Phys. Lett. 465, 57 (2008).

${ }^{80} \mathrm{G}$. Garcia-Belmonte, A. Munar, E. M. Barea, J. Bisquert, I. Ugarte, and R. Pacios, Org. Electron. 9, 847 (2008).

${ }^{81}$ T. Kirchartz, W. Gong, S. A. Hawks, T. Agostinelli, R. C. I. MacKenzie, Y. Yang, and J. Nelson, J. Phys. Chem. C 116, 7672 (2012).

${ }^{82}$ R. A. Street, Phys. Rev. B 82, 207302 (2010).

${ }^{83}$ C. Deibel and A. Wagenpfahl, Phys. Rev. B 82, 207301 (2010). 